\title{
Boiling induced atomization appeared in the liquid film by wall impinging jet on the superheated wall
}

\author{
Noritaka Sako*1, Jun Hayashi ${ }^{1}$, Chihiro Inoue ${ }^{2}$, Hiroshi Kawanabe ${ }^{1}$, Yu Daimon ${ }^{3}$ \\ ${ }^{1}$ Department of Energy Science, Kyoto University, Kyoto, Japan \\ ${ }^{2}$ Department of Aeronautics and Astronautics, Kyushu University, Fukuoka, Japan \\ ${ }^{3}$ Research and Development Directorate, Japan Aerospace Exploration Agency, Ibaraki, \\ Japan \\ *Corresponding author email : sako.noritaka.32c@st.kyoto-u.ac.jp
}

\begin{abstract}
Characteristics of boiling-induced atomization appeared in the liquid film which formed on the heated metal plate by a wall-impinging jet were experimentally investigated. The boilinginduced atomization were visualized via magnified high-speed imaging. Simultaneously, the variation of the wall temperature was measured by an infra-red camera from back side. The droplet size distribution and the ejection velocity distribution of the disintegrated small spherical particles were analysed. Results showed that two types of droplets were mainly observed in the high-speed magnified imaging. Those were the large droplet disintegrated from ligament observed in a region of relatively high superheat degree on the wall, and the small droplets from ligament formed in the collapse of the bubbles of nucleate boiling. Besides the ejection velocity of small spherical droplets was distributed around We $O(1)$.
\end{abstract}

\section{Keywords}

Liquid film, Boiling, Magnified imaging, Droplet size distribution, Theoretical analysis.

\section{Introduction}

Impinging jet cooling technique is widely used in the industrial fields such as material processing[1][2][3], cooling of nuclear reactor cores[4][5], bipropellant propulsion systems[6][7] and many more. In this cooling technique, liquid films are formed on the wall whose temperature exceeds the saturated temperature or the Leidenfrost temperature of the coolant. Boiling phenomena in the liquid film take a large amount of heat away from the wall. Additionally, most of the coolant is splashed away from the leading edge of the liquid film due to the vapor flow induced by vigorous boiling. The boundary of the wetting region and dry region is called as "wetting front (WF)"[8]. The WF moves downstream after the jet impingement. During the cooling process, the splashed droplets of the coolant atomization due to the boiling occurs in the liquid film.

It is important to know the characteristic values such as the ratio of the number of splashed droplets to the coolant flow rate in order to consider the flow and heat transfer characteristics of the liquid film on the heated surface. However, there is limited information on the boiling phenomena near the WF and the subsequent droplet formation processes in the liquid film formed by the impingement jet, while there have been researches on the behaviour of droplets impinging on the heated wall with atomization processes in the field of spray cooling and direct injection engines[9][10]. As the first step to clarify the atomization processes induced by the boiling phenomena, it is necessary to obtain experimental findings, such as the droplet diameter and velocity distribution.

In this study, therefore, boiling induced atomization processes appered in the liquid film by wall impinging jet on the superheated wall were visualized via magnified high-speed imaging to understand the boiling induced atomization. 


\section{Experimental apparatus and method}

Figure 1 shows a schematic of the experimental apparatus. In this paper, the point where the liquid jet impinged was defined as the origin, and the $x-y-z$ axes represent in the direction of the liquid film flow, liquid film width and perpendicular to the wall, respectively. The experimental apparatus consisted of an injector with a single nozzle, a coolant supply system, a metal plate (aluminium, $2 \mathrm{~mm}$ thick, $50 \mathrm{~mm}$ wide and $66 \mathrm{~mm}$ long), a rod heater used to rise the metal plate temperature, and a magnified high-speed imaging system. The test liquid was water at room temperature of approximately $20^{\circ} \mathrm{C}$. Bellows, lens (Nikon Zoom-Nikkor, focal length 35-70 mm, F\#3.3) and a high-speed camera (Photron FASTCAM SA1.1) were used for the magnified high-speed imaging with the backlight technique. The frame rate for the magnified imaging was set to $20000 \mathrm{fps}$. To visualise a series of atomization process in detail the magnified imaging with a higher frame rate of $54000 \mathrm{fps}$ was also performed. The exposure time for both imaging were set to $8.3 \mu \mathrm{s}$ and $5.4 \mu \mathrm{s}$ at the frame rate of $20000 \mathrm{fps}$ and of $54000 \mathrm{fps}$, respectively. The spatial resolution for magnified imaging was $5.74 \mu \mathrm{m} /$ pixel. Additionally, the change of the wall temperature distribution was measured by an infra-red camera (NIPPON AVIONICS, InfRec R550pro) from back side. The back side of the metal plate was coated with a black paint ensuring an emissivity of 0.94 . The frame rate of IR imaging was $60 \mathrm{fps}$. The mass flow rate of the test liquid, nozzle diameter and impingement angle which were parameters affecting the liquid film formation were respectively fixed at $5.0 \mathrm{~g} / \mathrm{s}$, $0.7 \mathrm{~mm}$ and $10^{\circ}$ because the objective of this study was to clarify the boiling-induced atomization appeared in the liquid film on the heated wall. Water was injected when the wall temperature at the impingement point of the liquid jet reached $250^{\circ} \mathrm{C}$. Experimental conditions and imaging conditions are listed in Table 1.

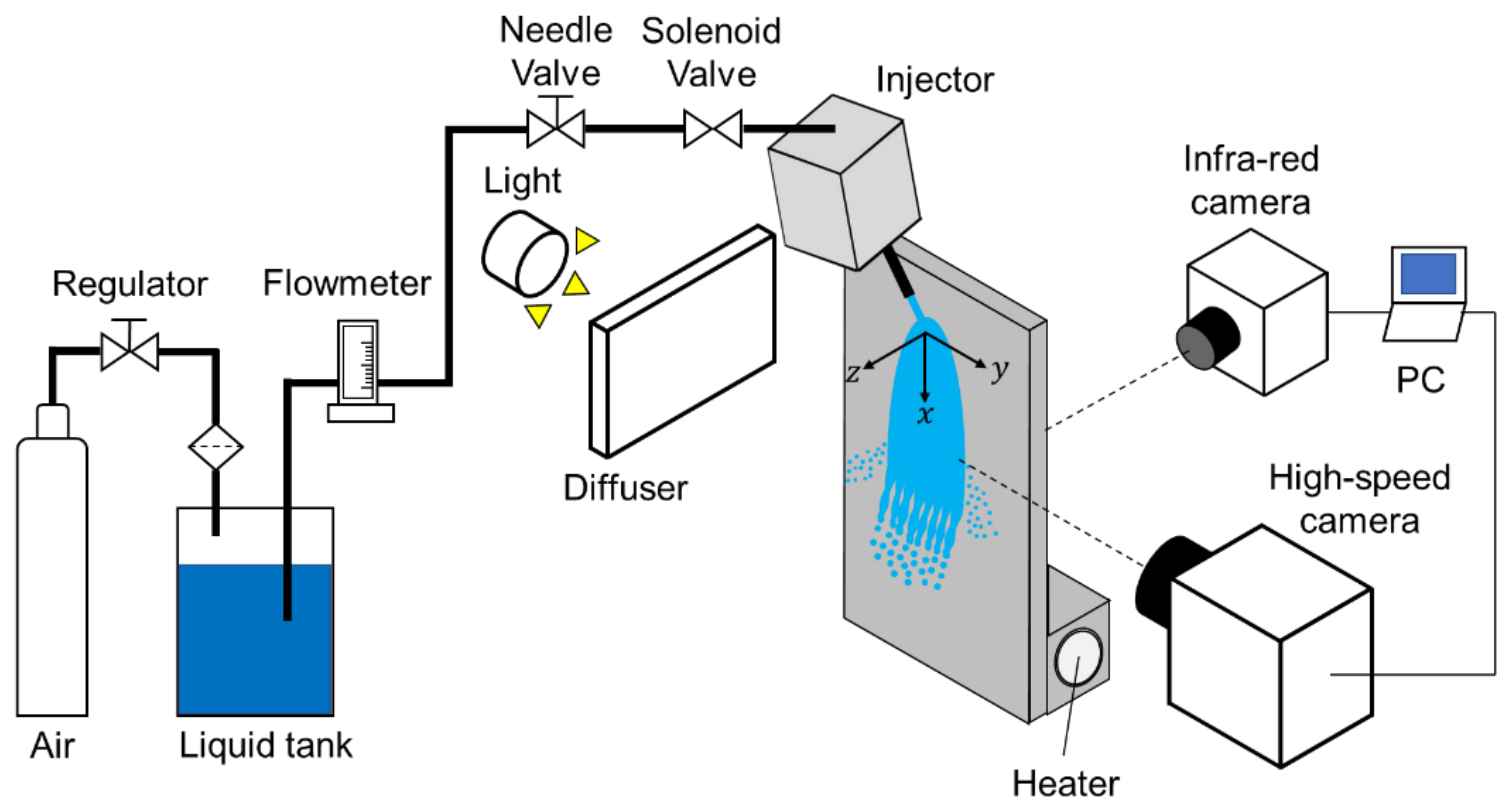

Figure 1. Schematic of experimental apparatus[11].

Table 1 - Experimental conditions and imaging conditions.

\begin{tabular}{cc|c}
\hline Mass flow rate of test liquid & {$[\mathrm{g} / \mathrm{s}]$} & 5.0 \\
\hline Nozzle diameter & {$[\mathrm{mm}]$} & 0.7 \\
\hline Impingement angle & {$[\mathrm{deg}]$.} & 10 \\
\hline Frame rate for magnified high-speed imaging & {$[\mathrm{fps}]$} & 20000,54000 \\
\hline Frame rate for IR imaging & {$[\mathrm{fps}]$} & 60 \\
\hline
\end{tabular}




\section{Results and Discussion}

Figure 2(a) and (b) show the top view and the side view of liquid film on the heated wall, respectively. As shown in Fig. 2(b), the liquid film was fully separated from the wall at the leading edge and the WF which was the boundary of the wetting region and dry region. In Fig. 2(b), it could be seen that many droplets dispersed from the wall both in the area upstream of the $W F((B)$ in Fig. 2(b)) and near the $W F((A)$ in Fig. 2(b)). As reported in many research [1-4], nucleate, transition and film boiling coexist in the liquid film during the quenching, and the droplets in Fig. 2 were considered to be caused by these boiling phenomena. Additionally, the size of droplets near the WF were larger than those in the area upstream of the WF.

Figure 3 shows direct images of a liquid film at different time and measurement positions for the magnified imaging. The liquid film expanded and the WF moved downstream with the elapsed time since the cooling was started as shown in Fig. 3. In this study, the position of imaging was fixed at $x=10 \mathrm{~mm}$. Two different timings from the start of cooling were selected to observe atomization processes both near the WF and in the area upstream of the WF. Those were the timing for observing the sequence processes of bubble growth, bursting, and atomization due to nucleate boiling, and that for the atomization at the rim of the liquid film. The visualized area at each imaging timing are shown in Fig. 3 as the yellow square. The images taken at the timing of Figs. 3(a) and (b) correspond to the atomization processes near the $W F((A)$ in Fig. 2(b)) and in the area upstream of the WF((B) in Fig. 2(b)), respectively. Besides, the change of wall superheat, which is defined as the difference between the wall temperature and the saturated temperature of the liquid, at each measurement point in Fig. 3(a) and (b) is shown in Fig. 4. As shown in Fig. 4, the wall superheat near the WF was relatively higher than that observed in upstream the WF due to the shorter time from the start of cooling. Figure 5(a) and (b) show the characteristic atomization processes observed at each timing in Figs. 3(a) and (b), in which the flow direction of the liquid film was from left side to right side. In Fig. 5(a), the atomization process on the wall with relatively higher wall superheat was observed, and it was clear from the images that the amplitude of the unstable wave on the ligament surface increased and the large droplet as seen in the region (A) of Fig. 2(b) pinched off from a ligament. On the other hand, it could be seen in Fig. 5(b) that bubble grew and burst. The small droplets disintegrated from the ligament and dispersed as shown in the region (B) of Fig. 2(b).

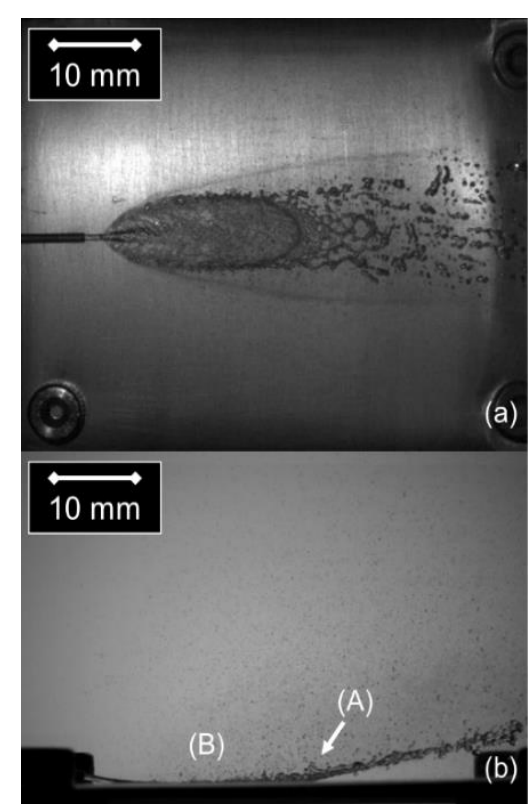

Figure 2. Direct image of liquid film formed on a hot wall; (a) from top of the liquid film, (b) from side of the liquid film[11].

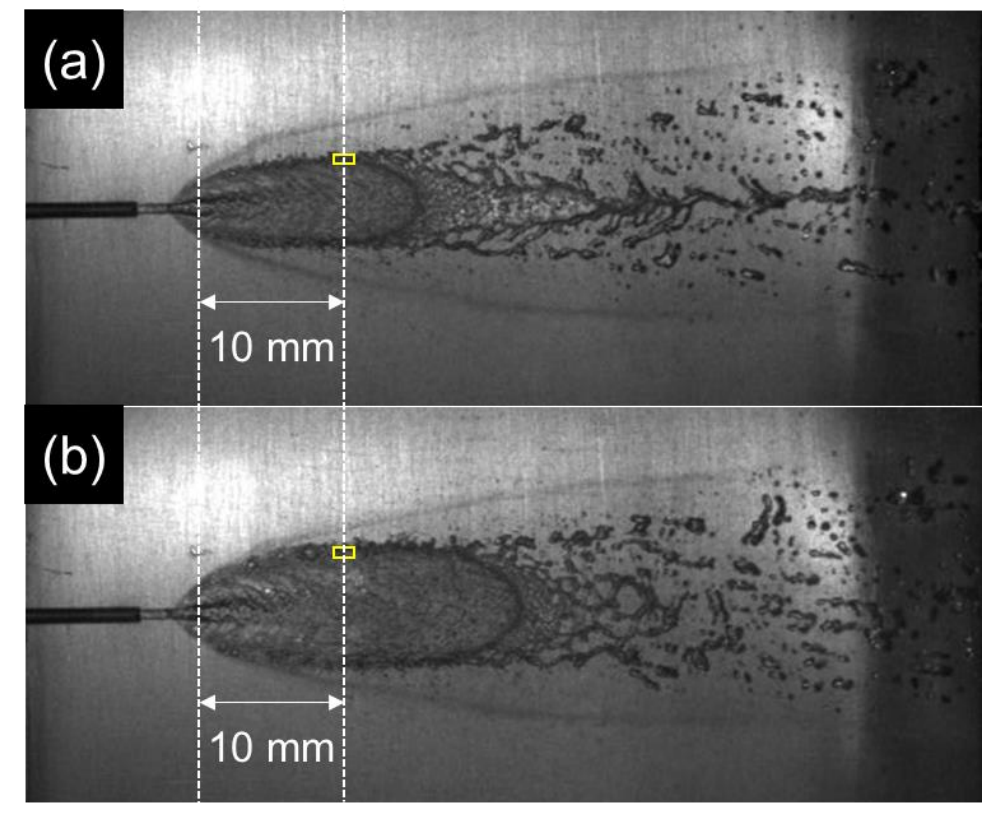

Figure 3. Measurement position on a liquid film (a) $0.30 \mathrm{~s}$ after start of injection, (b) $0.50 \mathrm{~s}$ after start of injection[11]. 

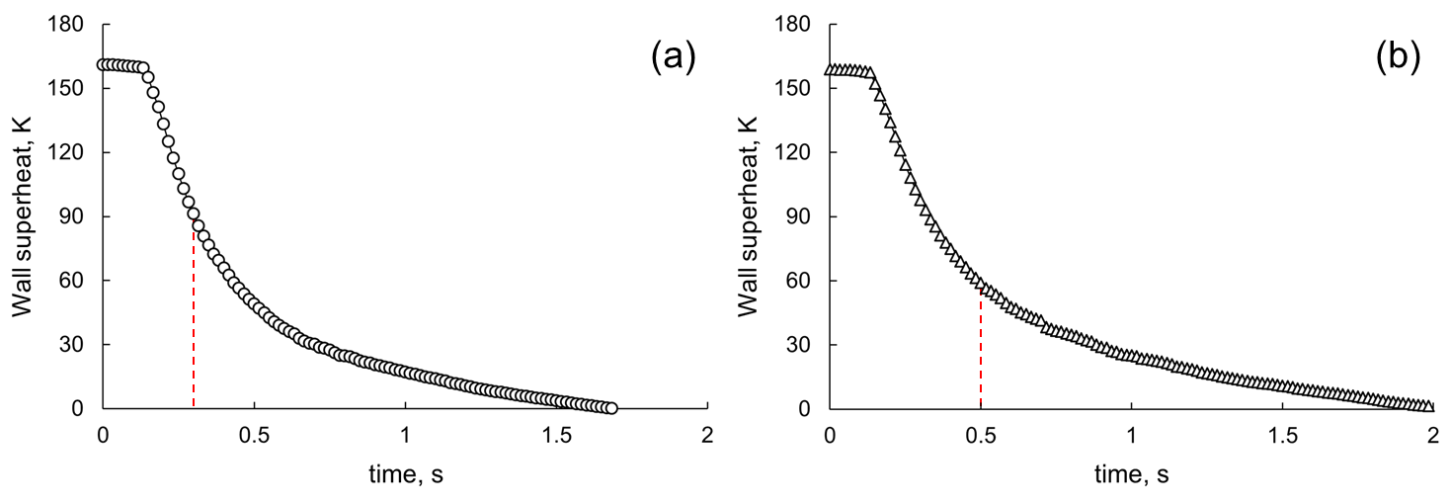

Figure 4. Change of wall superheat at each measurement position indicated in figure 3(a) and (b). Red dashed lines in (a) and (b) indicate $0.30 \mathrm{~s}$ and $0.5 \mathrm{~s}$ after start of injection, respectively.
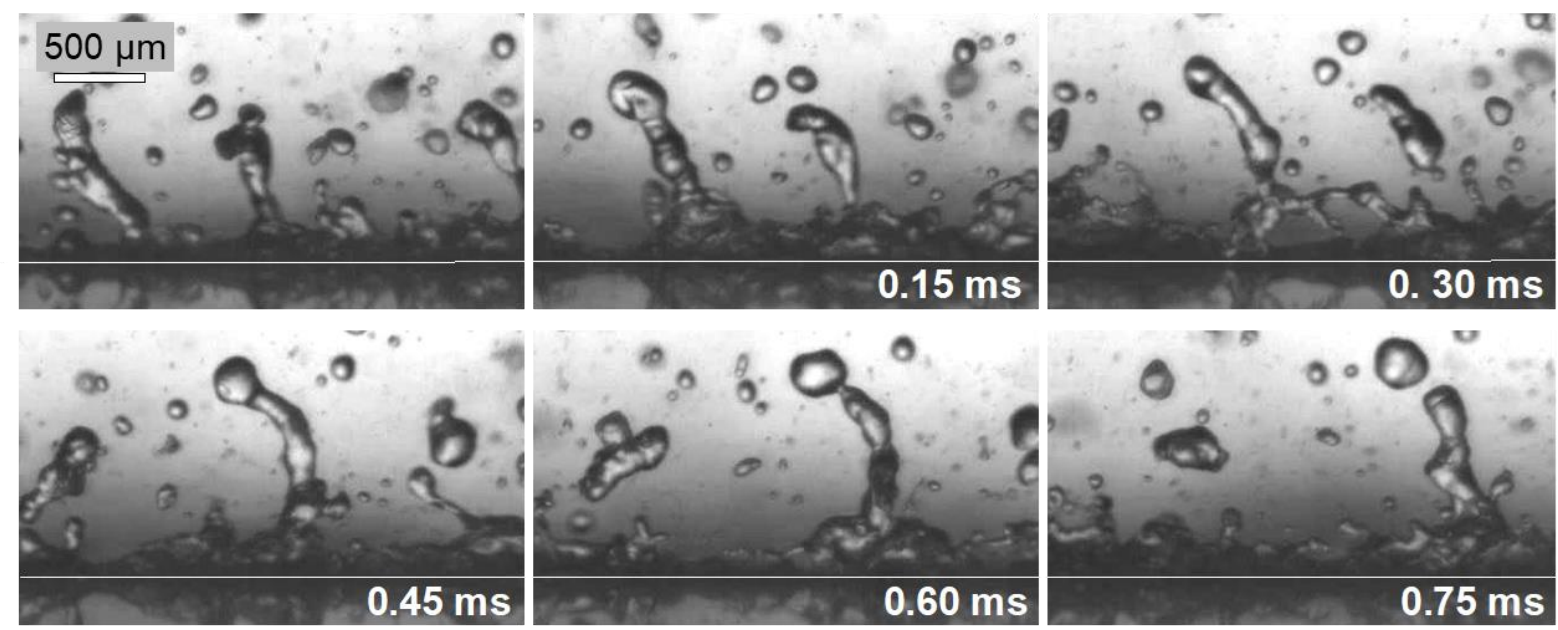

(a) Disintegration of droplets at the position near WF obtained at $0.3 \mathrm{~s}$ after start of injection
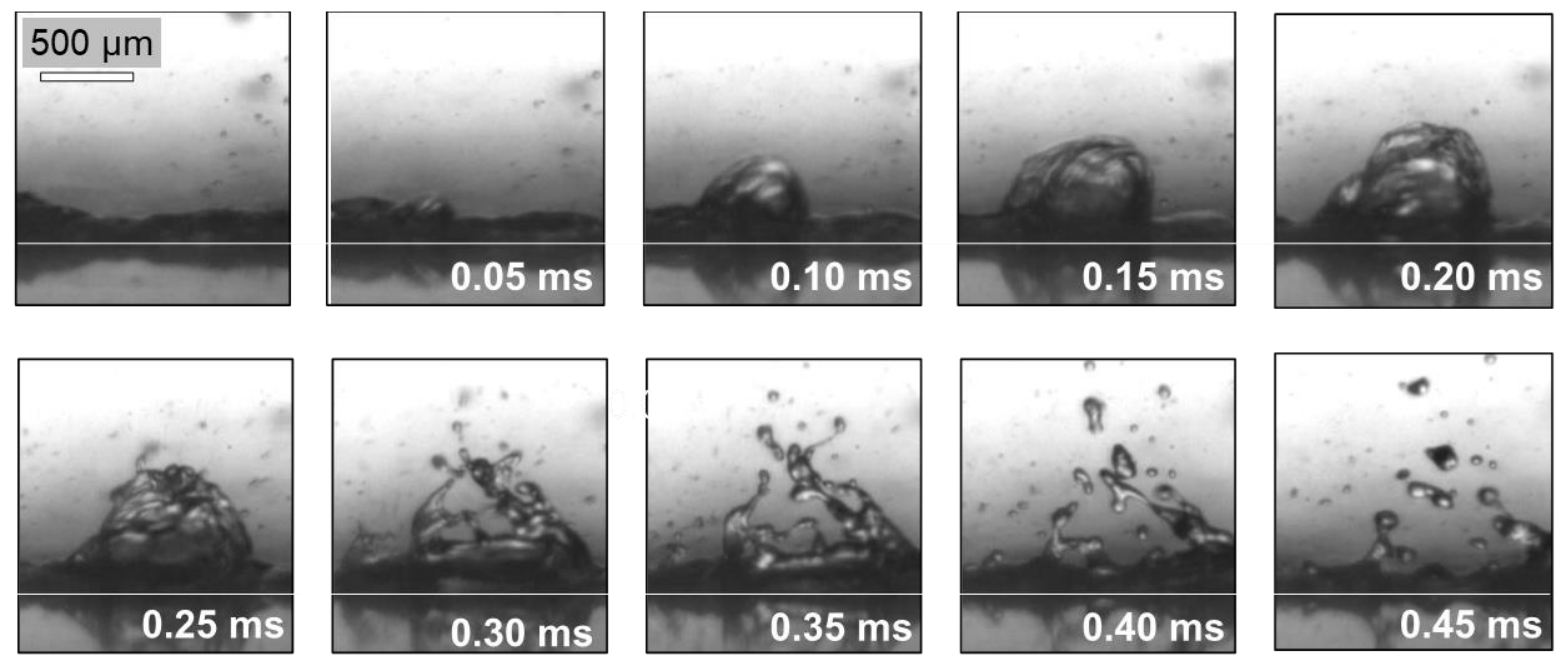

(b) Formation of droplets via collapse of bubble at the position away from WF obtained at $0.5 \mathrm{~s}$ after start of injection

Figure 5. Time series of boiling induced atomization at different timing[11].

To investigate the atomization process shown in Fig. $5(\mathrm{~b})$ in detail, a series of the process from bubble growth to disintegration was visualized at the frame rate of $54,000 \mathrm{fps}$. Initially, the growth process of the bubble was investigated. The bubble radius $b$ with the elapsed time since the bubble growth is confirmed to start is shown in Fig. 6 . The bubble radius was measured as the distance from the wall to the top of the bubble cap. As shown in Fig. 6, the 
bubble grew according to $b \sim\left(\alpha t^{*}\right)^{0.5}$ ( $\alpha$ : thermal diffusivity of liquid; $t^{*}$ : elapsed time since bubble start to growth), which meant that the bubble growth was governed by the heat transfer from the liquid phase to the gas phase, although it is not clear whether the bubble growth was dominated by the evaporation of superheated liquid surrounding the bubble[12] or the evaporating microlayer beneath the bubble[13]. After bursting the bubbles, no droplet formation or disintegration from the ligament with film retracting were observed as shown in Fig. 7. Generally, it is known that two kinds of droplets could be formed after bubble bursting; jet drops and film drops[14]. In this study, no jet drops were observed because only dry patch was formed on the wall after bubble bursting, while many film droplets as shown in Fig. 7(b) and (c) were observed. Here, the thickness of the bubble cap $h$ and droplet diameter $d$ from the bubble rupturing with one hole were considered. Figure 8 shows the relationship between the bubble radius just before rupture $R$ and the estimated value of $h$ calculated from Eq. (1) known as Taylor-Culick velocity[15][16] by measuring retraction speed;

$$
v=(2 \sigma / \rho h)^{0.5}
$$

where $\sigma$ and $\rho$ are the surface tension and density of the liquid. Although there was variation due to measurement from images (because the estimated value of $h$ is proportional to $v^{2}$ as

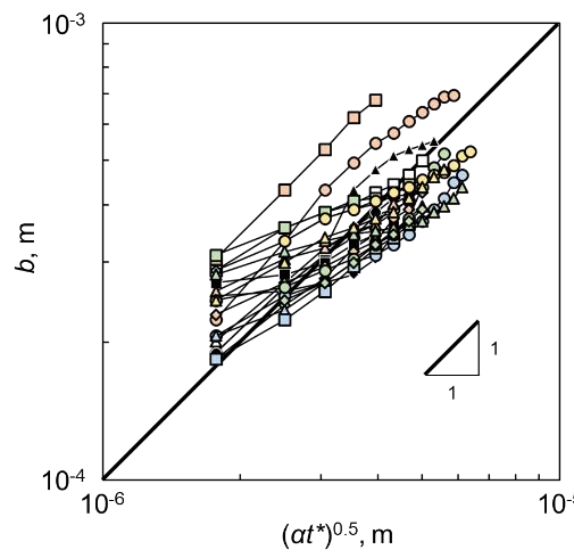

Figure 6. Growth process of bubble.
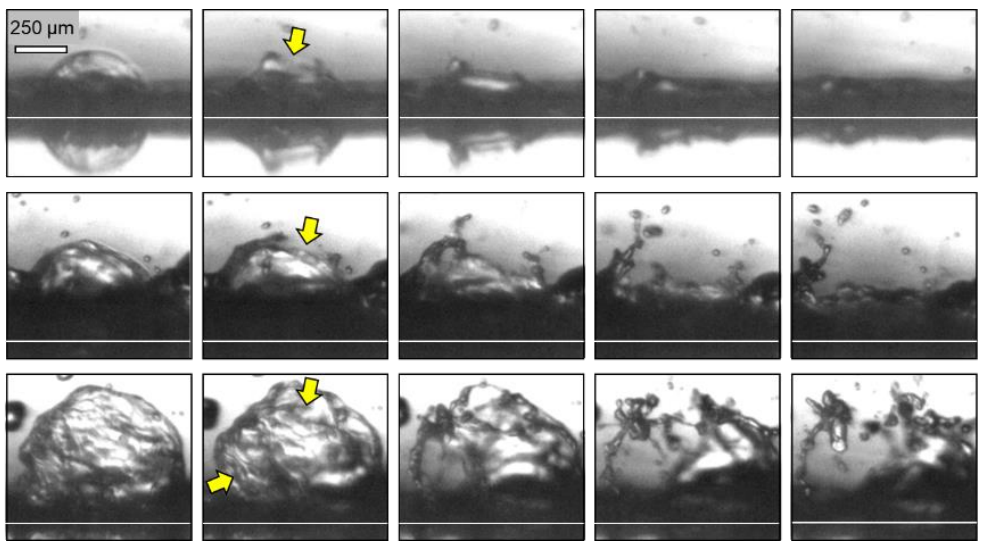

Figure 7. Time series after bubble bursting; no droplet formation(upper), rupturing with one hole(middle) and two holes(bottom). Yellow arrows indicate the position of holes.

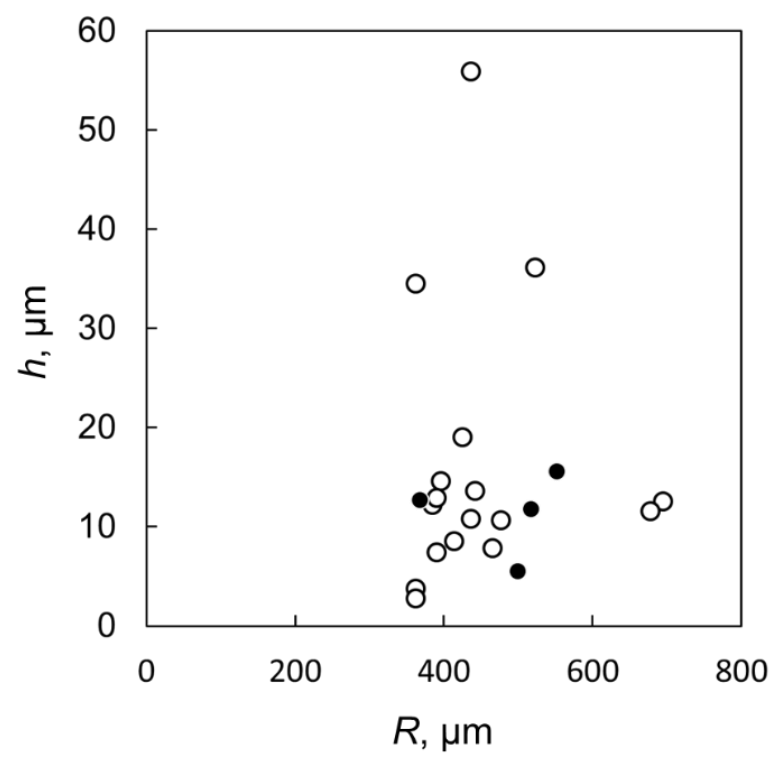

Figure 8. Estimated value of thickness of liquid film covering bubble. 
indicated in Eq.(1) [15][16]), it can be seen that $h \approx 10 \mu \mathrm{m}$ at any $R$. Then, the size of droplets from bursting bubbles was analysed although droplets that were in focus and clearly visible were able to be measured at only four of the results in Fig. 8 (shown as black plots). In the previous study, the mean droplet diameter $<d>$ from the bursting was scaled as Eq.(2) considering that a Rayleigh-Taylor instability induced by centripetal acceleration during retraction lead to the formation of ligaments which were then stretched out by centrifugation and the subsequent disintegration due to a Plateau-Rayleigh instability[17][18].

$$
<d>\sim R^{3 / 8} h^{5 / 8}
$$

Figure 9 shows the measurement result of the droplet diameter according to Eq.(2) using the estimated value of $h$. The droplet size of film drops may be well predicted by Eq.(2) although there were only four cases in which droplets were clearly visible to be measured.

To get overall aspects for boiling induced atomization processes, the droplet size and velocity distribution were analysed. For measuring the size of non-spherical droplets, the equivalent diameter which had the same area as projected area in obtained images was calculated. The distribution of the droplet size is shown in Fig. 10, and the Sauter mean diameter (SMD) $d_{32}$ defined as Eq.(3) was $206.2 \mu \mathrm{m}$.

$$
d_{32}=\Sigma d_{i}^{3} / \Sigma d_{i}^{2}
$$

$d_{i}$ is the diameter of each droplet. In Fig. 10, there are two groups of droplets those are the small droplets with a peak around $100 \mu \mathrm{m}$ and the relatively large size-distributed droplets around $200 \sim 300 \mu \mathrm{m}$. The smaller droplets were considered to be formed via collapse of the bubble as shown in Fig. 5(b). The larger droplets were thought to be formed on the wall with relatively high wall superheat where the transition boiling with the oscillation of solid-liquid contact occurred as shown in Fig. 5 (a). In such situation, the diameter of ligaments $d_{\mathrm{L}}$ could be considered to be approximately equal to the thickness of the liquid film $t$, and the droplet size pinched off from the ligament due to a Plateau-Rayleigh instability could be estimated using Eq.(4)[19].

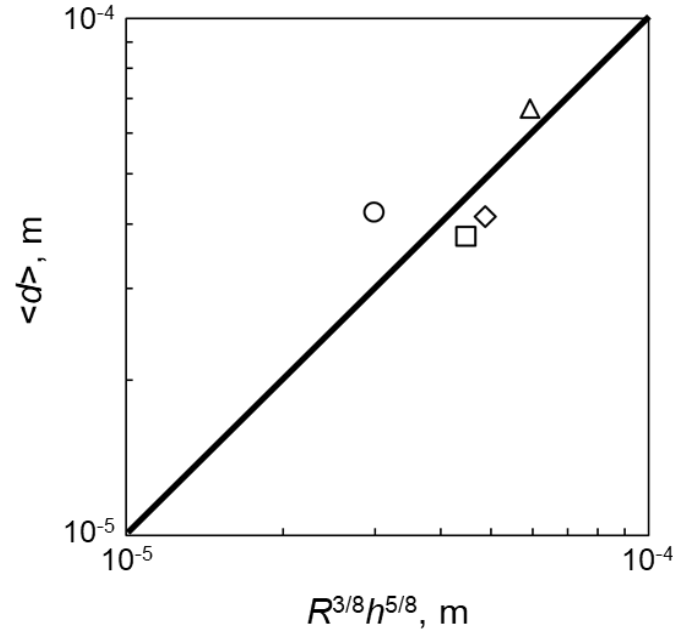

Figure 9. Mean diameter of some droplets from bursting bubble.

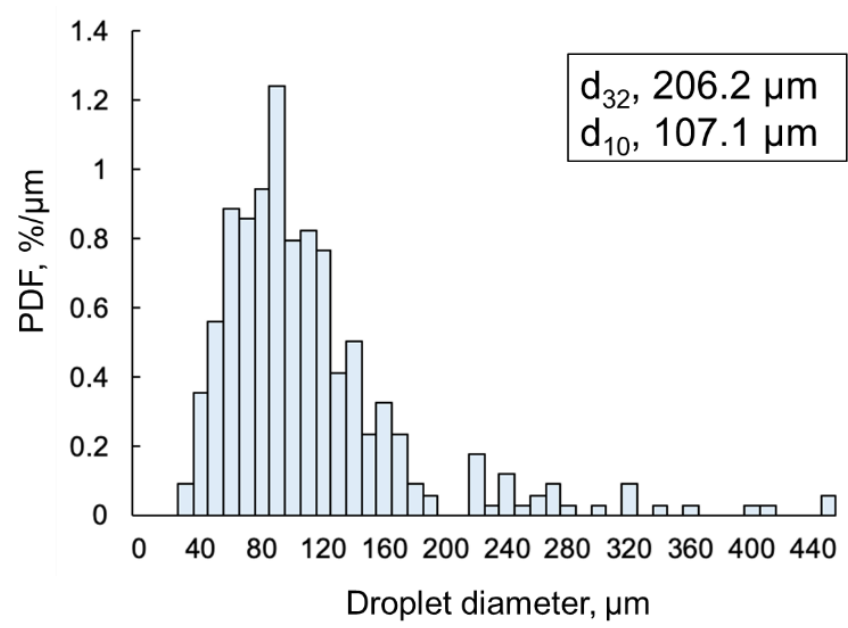

Figure 10. Droplet size distribution of disintegrated droplets. 


$$
d \approx 1.88 d\llcorner
$$

From Eq.(4), the size of droplets disintegrated on the relatively high temperature wall in this study was calculated to be about $200 \mu \mathrm{m}$ because the value of $t$ was approximately equal to $100 \mu \mathrm{m}$, and the calculated size of droplets was roughly consistent with the result of figure 10, which suggest that the droplet size disintegrated near the WF may depend on the thickness of the liquid film $t$.

Besides a scatter plot of the velocity of each droplet in $z$ direction versus droplet diameter is shown in Fig. 11. For the measurement of the velocity, only spherical droplets were analyzed for facilitation of the analysis. The bold line in Fig. 11 indicates the local Weber number defined as Eq.(5) of unity;

$$
W e=\rho U_{\mathrm{n}}^{2} d / \sigma
$$

where $U_{n}$ is the velocity of droplet in $z$ direction. As shown in figure $11, U_{n}$ was distributed around the local $W e \sim O(1)$, which indicate that the ejection velocity of droplets was defined by the relationship between the inertia of the ligament and the surface tension, and the disintegration from the ligament occurred around the local $W e \sim O(1)$.

\section{Summary and Conclusions}

To understand the characteristics of boiling induced atomization appeared in the liquid film by a wall-impinging jet on the superheated wall, atomization processes were visualized via magnified high-speed imaging with the backlight technique. The main findings in the present study are listed as follows.

1. Two types of droplets were mainly observed. Those were the large droplet disintegrated from ligament on the relatively high temperature wall, and the small droplets from ligament formed via the collapse of bubbles.

2. The heat-transfer-governed bubble growth and the subsequent disintegration process with bubble bursting were observed.

3. The ejection velocity of small spherical droplets was distributed around the local We $\sim O(1)$ and defined by the relationship between the inertia of the ligament and the surface tension.

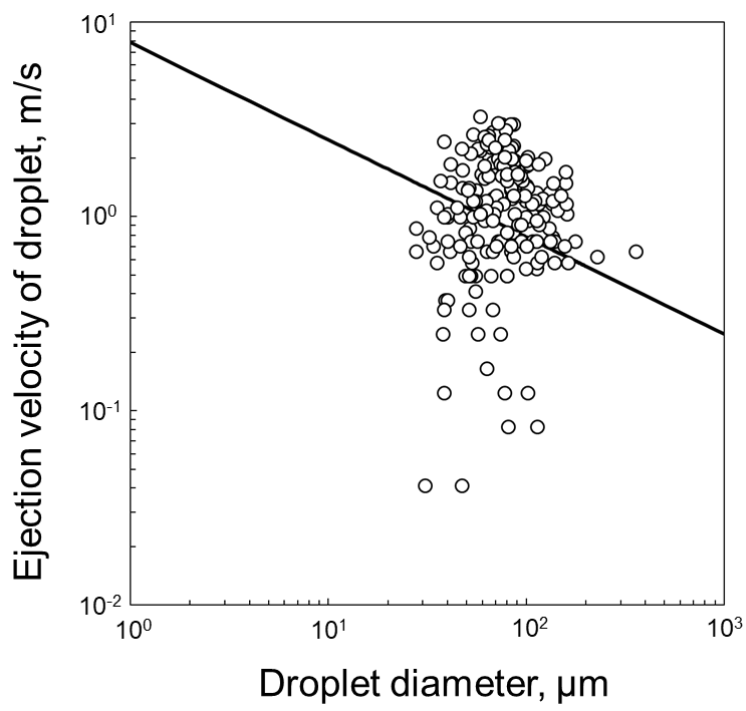

Figure 11. Relation between ejection velocity and droplet diameter[11]. 


\section{Acknowledgments}

We would like to acknowledge help for data analysis with Masaharu Nakajima.

\section{References}

[1] B. Wang, D. Lin, Q. Xie, Z. Wang, and G. Wang, "Heat transfer characteristics during jet impingement on a high-temperature plate surface," Appl. Therm. Eng., vol. 100, pp. 902-910, 2016.

[2] S. G. Lee, M. Kaviany, C. J. Kim, and J. Lee, "Quasi-steady front in quench subcooled-jet impingement boiling: Experiment and analysis," Int. J. Heat Mass Transf., vol. 113, pp. 622634, 2017.

[3] J. Hammad, Y. Mitsutake, and M. Monde, "Movement of maximum heat flux and wetting front during quenching of hot cylindrical block," Int. J. Therm. Sci., vol. 43, no. 8 SPEC. ISS., pp. 743-752, 2004.

[4] A. K. Sharma, U. K. Lodhi, G. Kumar, and S. K. Sahu, "Effect of Jet Inclination and Coolant Flow Rate on Thermal and Rewetting Behavior during Bottom Jet Impingement on Hot Horizontal Surfaces," Steel Res. Int., vol. 90, no. 10, pp. 1-17, 2019.

[5] T. Okawa, K. Yamagata, and Y. Umehara, "Measurement of heat transfer coefficient profile during quenching of a vertical hot wall with a falling liquid film," Nucl. Eng. Des., vol. 363, no. December 2019, p. 110629, 2020.

[6] G. Fujii, Y. Daimon, C. Inoue, D. Shiraiwa, N. Tanaka, and K. Furukawa, "Visualization of pulse firing mode in hypergolic bipropellant thruster," J. Propuls. Power, vol. 36, no. 5, pp. 671-684, 2020.

[7] N. Sako, J. Hayashi, Y. Daimon, H. Tani, and H. Kawanabe, "Experimental analysis of the spreading of a liquid film on a bipropellant thruster chamber wall," vol. 16, no. 1, pp. 1-11, 2021.

[8] Y. Mitsutake and M. Monde, "Heat transfer during transient cooling of high temperature surface with an impinging jet," Heat Mass Transf. und Stoffuebertragung, vol. 37, no. 4-5, pp. 321-328, 2001.

[9] G. E. Cossali, M. Marengo, and M. Santini, "Thermally induced secondary drop atomisation by single drop impact onto heated surfaces," Int. J. Heat Fluid Flow, vol. 29, no. 1, pp. 167-177, 2008.

[10] A. Sofia and M. ÆE. Anto, "Development of empirical correlations to predict the secondary droplet size of impacting droplets onto heated surfaces," pp. 755-768, 2009.

[11] N. Sako, J. Hayashi, C. Inoue, H. Kawanabe, and Y. Daimon, "Nucleate boiling induced atomization appeared in the thin liquid film on the heated solid wall," Proc. 29th ILASS-Japan Symp., 2020.

[12] M. S. Plesset and S. A. Zwick, "The Growth of Vapor Bubbles in Superheated Liquids," J. Appl. Phys., vol. 25, no. 4, pp. 493-500, 1954.

[13] M. G. Cooper, "The microlayer and bubble growth in nucleate pool boiling," Int. J. Heat Mass Transf., vol. 12, no. 8, pp. 915-933, 1969.

[14] D. C. Blanchard and L. D. Syzdek, "Film drop production as a function of bubble size," $J$. Geophys. Res., vol. 93, no. C4, pp. 3649-3654, 1988.

[15] F. E. C. Culick, "Comments on a Ruptured Soap Film," J. Appl. Phys., vol. 31, no. 6, pp. 1128$1129,1960$.

[16] A. B. Pandit and J. F. Davidson, "Hydrodynamics of the rupture of thin liquid films," J. Fluid Mech., vol. 212, pp. 11-24, 1990.

[17] H. Lhuissier and E. Villermaux, "Bursting bubbles," Phys. Fluids, vol. 21, no. 9, pp. 1-2, 2009.

[18] H. Lhuissier and E. Villermaux, "Bursting bubble aerosols," J. Fluid Mech., vol. 696, pp. 5-44, 2012.

[19] N. Dombrowski and P. C. Hooper, "The effect of ambient density on drop formation in sprays," Chem. Eng. Sci., vol. 17, no. 4, pp. 291-305, 1962. 